\title{
NUMERICAL SOLUTION OF BOOLE'S RULE IN NUMERICAL INTEGRATION BY USING GENERAL QUADRATURE FORMULA
}

\author{
P. V.Ubale \\ Associate professor in statistics \\ G.S.College of Science, Arts, Comm. \\ Khamgaon 444303 \\ Email: - prafullaubale@hotmail.com
}

Keywords: Numerical integration, Boole's rule, Classical quadrature formula

\begin{abstract}
We have seen that definite integrals arise in many different areas and that the fundamental theorem of calculus is a powerful tool for evaluating definite integrals. This paper describes classical quadrature method for the numerical solution of Boole's rule in numerical integration.
\end{abstract}

\section{1) Introduction}

Numerical integration is the study of how the numerical value of an integral can be found. It is also called as quadrature which refers to finding a square whose area is the same as the area under the curve. The inverse process to differentiation in calculus is represented by

$$
I=\int_{a}^{b} f(x) d x
$$

Which means that the integral of a function $f(x)$ with respect to the independent variable $x$ evaluated between the limits $x=a$ to $x=b$.

Equation 1.1.1 also corresponds to the area under the curve of $f(x)$ between $x=a$ and $x=b$. This is better graphically illustrated in fig 1 .

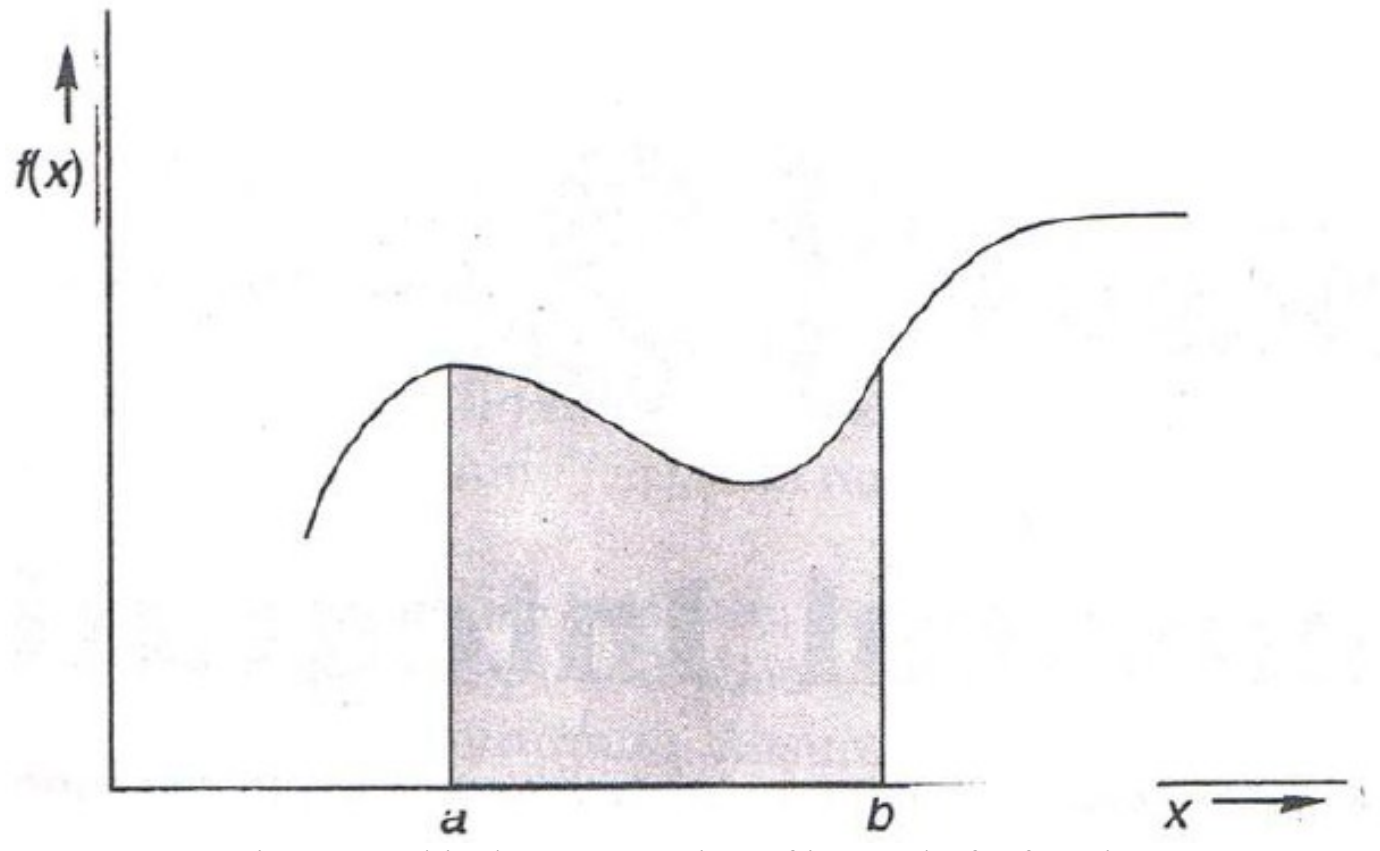

Fig. 1 Graphical representation of integral of a function

The problem of integration is simply reduced to the problem of finding shaded area. A better alternative approach could be to use a technique that uses simple arithmetic operations to compute area. This approach is called as numerical integration or numerical quadrature. 
Numerical integration method uses an interpolating polynomial $\mathrm{P}_{\mathrm{n}}(\mathrm{x})$ in the place of $\mathrm{f}(\mathrm{x})$ which can be integrated analytically.

Thus

$$
\mathrm{I}=\int_{a}^{b} f(x) d x=\int_{a}^{b} P_{n}(x) d x
$$

\section{2) A General Formula for solving Numerical Integration}

This formula is also called as general quadrature formula.

Suppose $f(x)$ is given for equidistant values of $x$ say $a=x_{0}, x_{0}+h, x_{0}+2 h, \ldots ., x_{0}+n h=b$. Let the range of integration $(a, b)$ is divided into $n$ equal parts each of width $h$ so that $b-a=n h$

By using fundamental theorem of numerical analysis it has been proved the general quadrature formula which is as follows.

$$
\begin{aligned}
\mathrm{I}=h[ & n f\left(x_{0}\right)+\frac{n^{2}}{2} \Delta f\left(x_{0}\right)+\left(\frac{n^{3}}{3}-\frac{n^{2}}{2}\right) \frac{\Delta^{2} f\left(x_{0}\right)}{2 !}+\left(\frac{n^{4}}{4}-n^{3}+n^{2}\right) \frac{\Delta^{3} f\left(x_{0}\right)}{3 !}+ \\
& \left.+\left(\frac{n^{5}}{5}-\frac{3}{2} n^{4}+\frac{11}{3} n^{3}-3 n^{2}\right) \frac{\Delta^{4} f\left(x_{0}\right)}{4 !}+\ldots . .+ \text { up to }(\mathrm{n}+1) \text { terms }\right]
\end{aligned}
$$

By putting $n$ to different values various formulae is used to solve numerical integration. They are

i) Trapezoidal rule

ii) Simpson's $1 / 3^{\text {rd }}$ rule

iii) Simpson's $3 / 8^{\text {th }}$ rule

iv) Boole's rule

In this paper we mainly focus on to demonstrating Boole's rule in numerical integration.

\section{3) Boole's rule in numerical integration}

Consider a function $\mathrm{y}=\mathrm{f}(\mathrm{x})$ over the interval $\left[\mathrm{x}_{0}, \mathrm{x}_{4}\right]$. Boole's rule is an approximation to the integral of $\mathrm{f}(\mathrm{x})$ over $\left[\mathrm{x}_{0}, \mathrm{x}_{4}\right]$. Boole's rule named after George Boole, a famous mathematician, is being derived by putting $\mathrm{n}=4$ in the general quadrature formula

$\mathrm{n}=4$ means $\mathrm{f}(\mathrm{x})$ can be approximated by a polynomial of $4^{\text {th }}$ degree so that fifth and higher order differences are vanishes in the general quadrature formula.

$\mathrm{I}=\int_{a}^{b} f(x) d x=\int_{x_{0}}^{x_{4}} f(x) d x=\int_{a}^{a+4 h} f(x) d x \quad$ where $\mathrm{x}_{\mathrm{i}}=\mathrm{a}+\mathrm{ih}$ and $\mathrm{h}=\frac{(b-a)}{4}$

Substituting $\mathrm{n}=4$ in the equation 1.2.1

$$
\begin{aligned}
\mathrm{I}=\int_{a}^{a+4 h} f(x) d x= & h\left[4 f(a)+8 \Delta f(a)+\left(\frac{64}{3}-8\right) \frac{\Delta^{2} f(a)}{2 !}+(64-64+16) \frac{\Delta^{3} f(a)}{3 !}\right. \\
& \left.+\left(\frac{1024}{5}-384+\frac{704}{3}-48\right) \frac{\Delta^{4} f(a)}{4 !}\right]
\end{aligned}
$$




$$
\begin{aligned}
& \mathrm{I}=\frac{h}{45}[14 f(a)+64 f(a+h)+24 f(a+2 h)+64 f(a+3 h)+14 f(a+4 h)] \\
& \mathrm{I}=\frac{2 h}{45}[7 f(a)+32 f(a+h)+12 f(a+2 h)+32 f(a+3 h)+7 f(a+4 h)] \\
& \mathrm{I}=\frac{2 h}{45}\left[7 f(a)+32 f\left(x_{1}\right)+12 f\left(x_{2}\right)+32 f\left(x_{3}\right)+7 f(b)\right]
\end{aligned}
$$

Equation 1.3.1 is the Boole's rule which represents area under the curve $y=f(x)$ and between the ordinates $\mathrm{x}=\mathrm{a}$ and $\mathrm{x}=\mathrm{b}$.

\section{4) Composite Boole's rule}

If the range of integration is from a to $\mathrm{a}+\mathrm{nh}=\mathrm{b}$ The Boole's rule can be improved by dividing the interval $(a, b)$ into small subintervals of width $4 \mathrm{~h}$ and apply Boole's rule for each of the subinterval. The sum of areas of all subintervals is the integral of the interval $(a, b)$. This is known as Composite Boole's rule.

$$
\begin{aligned}
\mathrm{I}= & \int_{a}^{a+n h} f(x) d x=\int_{x_{0}}^{x_{n}} f(x) d x=\int_{x_{0}}^{x_{4}} f(x) d x+\int_{x_{4}}^{x_{8}} f(x) d x+\ldots \ldots+\int_{x_{n-4}}^{x_{n}} f(x) d x \\
\mathrm{I}= & \int_{a}^{a+n h} f(x) d x=\int_{a}^{a+4 h} f(x) d x+\int_{a+4 h}^{a+8 h} f(x) d x+\ldots . .+\int_{a+(n-4)}^{a+n h} f(x) d x \\
\mathrm{I}= & \frac{2 h}{45}[7 f(a)+32 f(a+h)+12 f(a+2 h)+32 f(a+3 h)+7 f(a+4 h)] \\
& +\frac{2 h}{45}[7 f(a+4 h)+32 f(a+5 h)+12 f(a+6 h)+32 f(a+7 h)+7 f(a+8 h) \\
& +\ldots .+\frac{2 h}{45}[7 f(a+(n-4) h)+32 f(a+(n-3) h)+12 f(a+(n-2) h)+32 f(a+(n-1) h) \\
\mathrm{I}= & \frac{2 h}{45}\left[7\left\{f_{a}+f_{b}\right\}+32\left\{f_{1}+f_{3}+f_{5}+\cdots+f_{n-3}+f_{n-1}\right\}+12\left\{f_{2}+f_{6}+f_{10}+\cdots+f_{n-6}+f_{n-2}\right\}\right. \\
& \left.+14\left\{f_{4}+f_{8}+f_{12}+\cdots+f_{n-8}+f_{n-4}\right\}\right]
\end{aligned}
$$

\section{5) Application of Numerical Integration}

Numerical integration has always been useful in biostatistics to evaluate distribution function and other quantities. Emphasis in recent years on Bayesian and Empirical Bayesian method and on mixture models has greatly increased the importance of numerical integration for computing likelihood and posterior distributions and associated moments and derivatives. Many recent statistical methods are dependant especially on multiple integration possibly in very high dimension. 


\section{6) References}

[1] Casaletto J, Pickett M and Rice J. R. (1969): A comparison of some numerical integration programs, Signum Newsletter, 4, 30-40

[2] Kaishun Li, Peter Lumb (1985): Reliability analysis by numerical integration and curve fitting, Elsevier, vol.3, issue1, 29-36.

[3] Cools R (1992): A survey of methods for constructing cubature formulae in numerical integration, recent developments software and applications 1-24.

[4] Evans M and Swartz T (1995): Methods for approximating integrals in statistics with special emphasis on Bayesian integration problems, Statistical science,10,254-272.

[5] E Balagurusamy (1999): Numerical Methods Tata McGraw Hill Publishing Company Ltd.

[6] Devi Prasad (2003): an introduction to numerical analysis third edition, Narosa Publishing House. 